\title{
THE CYCLICITY OF POLYNOMIAL CENTERS VIA THE REDUCED BAUTIN DEPTH
}

\author{
ISAAC A. GARCÍA
}

(Communicated by Yingfei Yi)

\begin{abstract}
We describe a method for bounding the cyclicity of the class of monodromic singularities of polynomial planar families of vector fields $\mathcal{X}_{\lambda}$ with an analytic Poincaré first return map having a polynomial Bautin ideal $\mathcal{B}$ in the ring of polynomials in the parameters $\lambda$ of the family. This class includes the nondegenerate centers, generic nilpotent centers and also some degenerate centers. This method can work even in the case in which $\mathcal{B}$ is not radical by studying the stabilization of the integral closures of an ascending chain of polynomial ideals that stabilizes at $\mathcal{B}$. The approach is based on computational algebra methods for determining a minimal basis of the integral closure $\overline{\mathcal{B}}$ of $\mathcal{B}$. As far as we know, the obtained cyclicity bound is the minimum found in the literature.
\end{abstract}

\section{INTRODUCTION AND STATEMENT OF THE MAIN RESUlTS}

In this work we study families of real polynomial planar vector fields $\mathcal{X}_{\lambda}(x, y)=$ $A(x, y ; \lambda) \partial_{x}+B(x, y ; \lambda) \partial_{y}$, parameterized by its coefficients $\lambda \in \mathbb{R}^{M}$. Thus $A$ and $B$ are polynomials in the variables $x$ and $y$ and we collect all its coefficients in the parameter vector $\lambda$. As usual we associate to $\mathcal{X}_{\lambda}$ the planar polynomial differential system $\dot{x}=A(x, y ; \lambda), \dot{y}=B(x, y ; \lambda)$.

We assume that the full family $\mathcal{X}_{\lambda}$ possesses an isolated monodromic singularity at the fixed point $p_{0} \in \mathbb{R}^{2}$; hence $\mathcal{X}_{\lambda}\left(p_{0}\right)=0$ and nearby trajectories of $\mathcal{X}_{\lambda}$ rotate about $p_{0}$. We denote by $D \mathcal{X}\left(p_{0}\right)$ the linear part (Jacobian) of $\mathcal{X}$ at $p_{0}$. If the eigenvalues of $D \mathcal{X}\left(p_{0}\right)$ are nonzero pure imaginary, then $p_{0}$ is monodromic and it is either a nondegenerate center or a focus. When $D \mathcal{X}\left(p_{0}\right)$ is nonzero and has two zero eigevalues, then $p_{0}$ is called a nilpotent singularity and, under some specific extra assumptions (see [1]), it can also be monodromic. Finally, when $D \mathcal{X}\left(p_{0}\right)$ is zero, $p_{0}$ is termed degenerate, and in the particular case of having no characteristic direction it is also monodromic; see 6]. We emphasize that the fact that $p_{0}$ is monodromic for any $\lambda$ is very natural when it is nondegenerate since any unfolding can be put on this form but also that it is a strong restriction for the nilpotent and degenerate cases.

This paper is motivated by the so-called local sixteenth Hilbert problem for $\mathcal{X}_{\lambda}$ which asks for the cyclicity of $p_{0}$ in $\mathcal{X}_{\lambda}$, that is, to determine the maximum number of limit cycles (isolated periodic orbits) that can bifurcate from $p_{0}$ when

Received by the editors June 3, 2015 and, in revised form, July 3, 2015.

2010 Mathematics Subject Classification. Primary 37G15, 37G10, 34C07.

Key words and phrases. Center, polynomial vector fields, Bautin ideal, cyclicity, limit cycle.

The first author was partially supported by a MINECO grant number MTM2014-53703-P and by a CIRIT grant number 2014 SGR 1204. 
$p_{0}$ is monodromic for the full family. We call this number $\operatorname{Cyc}\left(\mathcal{X}_{\lambda}, p_{0}\right)$. More precisely, the cyclicity $\operatorname{Cyc}\left(\mathcal{X}_{\lambda}, p_{0}\right)$ of the monodromic singularity at $p_{0}$ of $\mathcal{X}_{\hat{\lambda}}$ is the maximum number of small amplitude limit cycles that can appear bifurcating from $p_{0}$ in family $\mathcal{X}_{\lambda}$ for $\|\lambda-\hat{\lambda}\| \ll 1$.

Using a transversal section $\Sigma=[0, \hat{h}(\lambda))$ to the local flow of $\mathcal{X}_{\lambda}$ with endpoint $h=0$ at $p_{0}$ and parameterized by $h$, we can define the displacement map $d$ : $\Sigma \times \mathbb{R}^{M} \rightarrow \Sigma$ defined by $d(h ; \lambda)=\Pi(h ; \lambda)-h$, where $\Pi$ is the Poincaré first return map which is (geometrically) well defined since $p_{0}$ is monodromic.

Throughout this work we will assume that the displacement map $d(h ; \lambda)$ is analytic in the variables $h \in[0, \hat{h})$ and $\lambda \in \mathbb{R}^{M}$. In particular $p_{0}$ can only be either a center or a focus. Moreover we can compute the Taylor series $d(h ; \lambda)=$ $\sum_{i \geq 1} v_{i}(\lambda) h^{i}$ about $h=0$. The coefficients $v_{i}$ are called Poincaré-Liapunov quantities. We also will assume that $v_{i} \in \mathbb{R}[\lambda]$. This framework is always true if the monodromic singularity at $p_{0}$ is either nondegenerate (see the book [11]) or nilpotent in generic cases (you can consult [7]) or degenerate in very special cases (see [5]).

Under the former assumptions, the Bautin ideal $\mathcal{B}$ in the polynomial ring $\mathbb{R}[\lambda]$ associated to the singularity $p_{0}$ of $\mathcal{X}_{\lambda}$ is defined as the ideal generated by all the polynomials $v_{i}(\lambda)$ with $i \in \mathbb{N}$. This ideal $\mathcal{B}$ is Noetherian, and then, by the Hilbert's basis theorem, it is generated by a finite number of polynomials; see for example [4. Let $k \in \mathbb{N}$ be the smallest positive integer such that $\mathcal{B}=\left\langle v_{1}(\lambda), v_{2}(\lambda), \ldots, v_{k}(\lambda)\right\rangle$. This number $k$ is called the Bautin index of $\mathcal{B}$ in 8 . In other words, we can define the ascending chain of ideals

$$
\mathcal{B}_{1} \subseteq \mathcal{B}_{2} \subseteq \cdots \subseteq \mathcal{B}_{k}=\mathcal{B}
$$

with $k$ the Bautin index of $\mathcal{B}$ and where we have defined the ideals $\mathcal{B}_{j}=\left\langle v_{1}(\lambda), \ldots\right.$, $\left.v_{j}(\lambda)\right\rangle$ for any integer $j \geq 1$.

A finite set of generators of a finitely generated ideal $\mathcal{J}$ is called a basis of $\mathcal{J}$. A basis of $\mathcal{J}$ said to be minimal if no proper subset of it is a basis of $\mathcal{J}$. Unfortunately, an ideal can have a minimal basis consisting of a different number of elements. Regarding the Bautin ideal $\mathcal{B}$, in this work we will use the notion of a minimal basis of $\mathcal{B}$ with respect to the ordered Poincaré-Liapunov quantities (and call it a minimal basis again omitting, for simplicity, any mention of an ordering). Now we give this definition and see from it that we attain uniqueness of the cardinality of this minimal basis.

Definition 1. Given the Bautin ideal $\mathcal{B}=\left\langle v_{i}(\lambda): i \in \mathbb{N}\right\rangle$, we say that the basis $B=\left\{v_{j_{1}}(\lambda), \ldots, v_{j_{m}}(\lambda)\right\}$ of $\mathcal{B}$ with order $1 \leq j_{1}<\cdots<j_{m}$ is minimal if it satisfies the following properties:

(i) $v_{i}(\lambda) \equiv 0$ for $1 \leq i \leq j_{1}-1$ and $a_{j_{1}}(\lambda) \not \equiv 0$.

(ii) For $i \geq j_{1}+1$, if $v_{i}(\lambda) \notin\left\langle v_{1}(\lambda), \ldots, v_{i-1}(\lambda)\right\rangle$, then $v_{i}(\lambda) \in B$.

Let $\left\{v_{j_{1}}(\lambda), v_{j_{2}}(\lambda), \ldots, v_{j_{m}}(\lambda)\right\}$ be a minimal basis of $\mathcal{B}$. The cardinality $m$ of this minimal basis is called the Bautin depth of $\mathcal{B}$ in [10, and clearly $m \leq k$.

If $\mathcal{X}_{\lambda^{\dagger}}$ has a focus at $p_{0}$, then $v_{1}\left(\lambda^{\dagger}\right)=\cdots=v_{r-1}\left(\lambda^{\dagger}\right)=0$ but $v_{r}\left(\lambda^{\dagger}\right) \neq 0$ for some index $r \geq 1$. We call $r$ the order of the focus $p_{0}$. In this situation, for $\lambda$ near $\lambda^{\dagger}$, the displacement map can be clearly written as $d(h ; \lambda)=\sum_{i=1}^{r-1} v_{i}(\lambda) h^{i}+$ $v_{r}(\lambda)[1+\psi(h, \lambda)] h^{r}$ with some analytic function $\psi$. From this we can deduce that $r-1$ or $r$ (depending on whether $p_{0}$ is degenerate or not respectively) is an upper bound for the cyclicity $\operatorname{Cyc}\left(\mathcal{X}_{\lambda}, p_{0}\right)$ of this focus; see for example Proposition 6.1.2 
in [1]. Of course this bound can be improved. This is the usual way in which the multiple Andronov-Hopf bifurcation of small amplitude limit cycles from a focus occurs.

On the contrary, $\mathcal{X}_{\lambda^{*}}$ has a center at $p_{0}$ if and only if $v_{i}\left(\lambda^{*}\right)=0$ for all $i \in \mathbb{N}$, and, in this case, for $\lambda$ near $\lambda^{*}$ the displacement map can be expressed as $d(h, \lambda)=$ $\sum_{j=1}^{m} v_{i_{j}}(\lambda)\left[1+\psi_{j}(h, \lambda)\right] h^{i_{j}}$, where $m$ is the Bautin depth of $\mathcal{B},\left\{v_{i_{1}}, \ldots, v_{i_{m}}\right\}$ is a minimal basis of $\mathcal{B}$, and $\psi_{j}$ are certain analytic functions; see [11,12] and the seminal paper 2]. Now after a repeated application of a Rolle's Theorem kind of argument (see Lemma 6.1.6 and Theorem 6.1.7 of [11]), it is proved that the cyclicity $\operatorname{Cyc}\left(\mathcal{X}_{\lambda}, p_{0}\right)$ of the center at $p_{0}$ of $\mathcal{X}_{\lambda^{*}}$ is bounded by $m-1$.

Definition 2. Let $R$ be an arbitrary Noetherian ring and $\mathcal{J}$ an ideal in $R$. An element $r \in R$ is said to be integral over $\mathcal{J}$ if there exist $s \in \mathbb{N}$ and elements $b_{i} \in \mathcal{J}^{i}$ such that $r^{s}+b_{1} r^{s-1}+b_{2} r^{s-2}+\cdots+b_{s}=0$. The set of all elements of $R$ that are integral over $\mathcal{J}$ is called the integral closure of $\mathcal{J}$ and is denoted by $\overline{\mathcal{J}}$. It follows that $\overline{\mathcal{J}}$ is an ideal and that $\mathcal{J} \subseteq \overline{\mathcal{J}} \subseteq \sqrt{\mathcal{J}}$, with $\sqrt{\mathcal{J}}=\left\{r \in R: r^{n} \in \mathcal{J}\right.$ for some $\left.n \in \mathbb{N}\right\}$ being the radical of $\mathcal{J}$. Finally, $\mathcal{J}$ is called radical if $\mathcal{J}=\sqrt{\mathcal{J}}$.

Now it is worth emphasizing the work [8]. There the authors introduce the termed reduced Bautin index $\bar{k}$ of $\mathcal{B}$ and they prove that $\operatorname{Cyc}\left(\mathcal{X}_{\lambda}, p_{0}\right) \leq \bar{k}$ when $p_{0}$ is a nondegenerate singularity. To be precise, $\bar{k}$ is the smallest integer such that the integral closure $\overline{\mathcal{B}}_{\bar{k}}$ of $\mathcal{B}_{\bar{k}}$ is just $\overline{\mathcal{B}}$, the integral closure of the Bautin ideal $\mathcal{B}$. More specifically $(8)$ consider the ascending chain of integral closures of the ideals $\mathcal{B}_{j}$, that is, $\overline{\mathcal{B}}_{1} \subseteq \overline{\mathcal{B}}_{2} \subseteq \cdots \subseteq \overline{\mathcal{B}}_{\bar{k}}=\overline{\mathcal{B}}$, so that $\bar{k}$ is just its moment of stabilization. This idea has merit since it is much easier to determine whether ideals have the same integral closure than to check whether they are equal. The recent books 9 and [13] deal with integral closures, emphasizing on integral closures of ideals. Notice that, since $\mathcal{B}_{j} \subseteq \overline{\mathcal{B}}_{j}$ for any $j \in \mathbb{N}$, one has $\bar{k} \leq k$, and therefore the cyclicity upper bound given by the reduced Bautin index is better than that obtained by the Bautin index.

Remark 3. There are two implementations for computing the integral closure of an ideal. One of them is normalI in the library reesclos. lib of Singular. The other option is integralClosure in the IntegralClosure package of MACAULAY2.

Clearly there is a minimal basis $\left\{v_{j_{1}}(\lambda), \ldots, v_{j_{\kappa}}(\lambda)\right\} \subset \mathbb{R}[\lambda]$ of $\mathcal{B}_{\bar{k}}$ formed by Poincaré-Liapunov quantities. We define its cardinal since it will be key in this work.

Definition 4. We call the reduced Bautin depth $\kappa \in \mathbb{N}$ of the Bautin ideal $\mathcal{B}$ the cardinality of a minimal basis of the ideal $\mathcal{B}_{\bar{k}}$ where $\bar{k}$ is the reduced Bautin index of $\mathcal{B}$.

The objective in this paper is to extend the ideas introduced in [8] to some nilpotent and degenerate monodromic singularities and also to improve all the known upper bounds of $\operatorname{Cyc}\left(\mathcal{X}_{\lambda}, p_{0}\right)$ by means of the reduced Bautin depth $\kappa$ of $\mathcal{B}$. Below we state our main results concerning the cyclicity problem and the approach to that problem using methods from computational commutative algebra.

Theorem 5. Let $\kappa$ be the reduced Bautin depth of the Bautin ideal $\mathcal{B}$ associated to a monodromic simgularity $p_{0}$ of family $\mathcal{X}_{\lambda}$. Then $\operatorname{Cyc}\left(\mathcal{X}_{\lambda}, p_{0}\right) \leq \kappa-1$. 
Notice how the cyclicity upper bound obtained in Theorem 5 improves the results obtained in the literature of the subject since

$$
\kappa \leq \bar{k} \text { and } \kappa \leq m \text {. }
$$

The first inequality comes from Definition 4 , whereas the second one is a direct consequence of the fact that $\bar{k} \leq k$ and Definition 1.

For a field $\mathbb{K}$ (here it will be either $\mathbb{K}=\mathbb{R}$ or $\mathbb{K}=\mathbb{C}$ ) we denote by $\mathbf{V}_{\mathbb{K}}(\mathcal{J}) \subset \mathbb{K}^{M}$ the affine variety associated to a polynomial ideal $\mathcal{J}=\left\langle p_{1}(\mathbf{x}), \ldots, p_{r}(\mathbf{x})\right\rangle$ in $\mathbb{K}[\mathbf{x}]$ with $\mathbf{x} \in \mathbb{K}^{M}$. Therefore $\mathbf{V}_{\mathbb{K}}(\mathcal{J})$ is the set of common zeros in $\mathbb{K}^{M}$ of all elements of $\mathcal{J}$; see [4].

To solve the center problem of the polynomial family $\mathcal{X}_{\lambda}$ at the monodromic singularity $p_{0}$ means to find the so-called center variety $\mathbf{V}_{\mathbb{R}}(\mathcal{B})$. In other words, $\mathcal{X}_{\lambda^{*}}$ has a center at $p_{0}$ if and only if $\lambda^{*} \in \mathbf{V}_{\mathbb{R}}(\mathcal{B}) \subset \mathbb{R}^{M}$. Notice that it may happen that $\mathbf{V}_{\mathbb{R}}(\mathcal{B})=\mathbf{V}_{\mathbb{R}}\left(\mathcal{B}_{r}\right)$ for some integer $r$, but $\mathbf{V}_{\mathbb{C}}(\mathcal{B}) \neq \mathbf{V}_{\mathbb{C}}\left(\mathcal{B}_{r}\right)$, where $\mathbf{V}_{\mathbb{R}}(\mathcal{B})=\mathbf{V}_{\mathbb{C}}(\mathcal{B}) \cap \mathbb{R}^{M}$.

In the particular case that $\overline{\mathcal{B}}$ is a radical ideal we can algorithmically find the reduced Bautin depth $\kappa \in \mathbb{N}$ of $\mathcal{B}$ as follows, provided that we have already solved the center problem (in the complex parameter setting) of family $\mathcal{X}_{\lambda}$.

Theorem 6. Let $\left\{v_{j_{1}}(\lambda), \ldots, v_{j_{\tau}}(\lambda)\right\}$ be a minimal basis of the ideal $\mathcal{B}_{j_{\tau}}$. Assume that the equality of varieties $\mathbf{V}_{\mathbb{C}}(\mathcal{B})=\mathbf{V}_{\mathbb{C}}\left(\mathcal{B}_{j_{\tau}}\right)$ holds in $\mathbb{C}^{M}$ where $\mathcal{B}$ is the Bautin ideal associated to a monodromic simgularity $p_{0}$ of family $\mathcal{X}_{\lambda}$. If $\overline{\mathcal{B}}_{j_{\tau}}=\sqrt{\overline{\mathcal{B}}_{j_{\tau}}}$, then $\tau=\kappa$, the reduced Bautin depth of $\mathcal{B}$, and, in particular, $\operatorname{Cyc}\left(\mathcal{X}_{\lambda}, p_{0}\right) \leq \kappa-1$.

\section{Proof of Theorem 5}

2.1. Preliminaries. First we recall the following Rouché theorem; see for example [3. This will be key in the proof of Theorem 5.

Theorem 7 (Rouché's theorem). Let $D \subset \mathbb{C}$ be a simply connected (open) domain with boundary $\partial D$ given by a closed simple path. If the complex-valued functions $f$ and $g$ are holomorphic on $D \cup \partial D$ and $|g(z)|<|f(z)|$ at each point $z \in \partial D$, then $f$ and $f+g$ have the same number of zeros on $D$, counting multiplicities.

2.2. Proof of Theorem [5. First we recall the main result of 8 ] and we state it using our notation. Let $d(h ; \lambda)=\sum_{i \geq 1} v_{i}(\lambda) h^{i}$ be the displacement map and let $\bar{k}$ be the reduced Bautin index of $\mathcal{B}$; hence $\overline{\mathcal{B}}_{\bar{k}}=\overline{\mathcal{B}}$. Then, in [8] it is proved that in a neighborhood of $h=0, d(h ; \lambda)$ has at most $\bar{k}$ distinct zeroes when $\left\|\lambda-\lambda^{*}\right\| \ll 1$ and $\lambda^{*} \in \mathbf{V}_{\mathbb{R}}(\mathcal{B})$. The main idea of that proof is first to complexify the displacement map. Hence $d(h ; \lambda)$ is considered with $h \in \mathbb{C}$ near $h=0$ and next to split $d(h ; \lambda)=$ $f(h ; \lambda)+g(h ; \lambda)$ with $f(h ; \lambda)=\sum_{i=1}^{\bar{k}} v_{i}(\lambda) h^{i}$ and $g(h ; \lambda)=\sum_{i>\bar{k}} v_{i}(\lambda) h^{i}$ for a further application of Rouché's theorem in order to bound the number of zeros of $d(h ; \lambda)$ in some disk $D$ centered at $h=0$. Actually, in $[8$ it is proved that, for a fixed $\lambda$ sufficiently close to $\lambda^{*}$, Rouché's theorem can be applied with the former split on $\partial D=\{h \in \mathbb{C}:|h|=R\}$ with a radius $R$ between $\eta(\bar{k}) R^{*}<R<R^{*}$, with $R^{*}$ the radius of convergence of the complex displacement map and $\eta(\bar{k})=5^{-2(\bar{k}+1)}$. In particular, since $f$ is a polynomial of degree $\bar{k}$, this implies that the number of zeroes of $d(h ; \lambda)$ on $D$ is at most $\bar{k}$, finishing the proof.

But in fact the former proof gives us more information: when $\lambda$ is close to $\lambda^{*}$, the number of zeroes of $d(h ; \lambda)$ on $D$ and the number of zeroes of $f(h ; \lambda)$ on $D$ coincide counting multiplicities. 
Let $\left\{v_{i_{1}}(\lambda), \ldots, v_{i_{\kappa}}(\lambda)\right\}$ with $\kappa \leq \bar{k}$ be a minimal basis of the ideal $\mathcal{B}_{\bar{k}}=$ $\left\langle v_{1}, \ldots, v_{\bar{k}}\right\rangle$. By the definition of minimal basis $1 \leq i_{1}<i_{2}<\cdots<i_{\kappa}$ so that we can rearrange $f(h ; \lambda)$ as

$$
f(h ; \lambda)=\sum_{j=1}^{\kappa} v_{i_{j}}(\lambda) h^{i_{j}}\left(1+P_{j}(h ; \lambda)\right),
$$

where $P_{j}(h ; \lambda)$ are polynomials in both variables $h$ and $\lambda$ such that $P_{j}(0 ; \lambda)=0$. Therefore the function $f$ behaves like a polynomial in $h$ of degree $\kappa$ near $\lambda=\lambda^{*}$, and hence can have at most $\kappa-1$ isolated positive zeros for any $\lambda$ in a neighborhood of $\lambda^{*}$ as it is proved in Proposition 6.1.2 of [11].

\section{Proof of Theorem 6}

3.1. Preliminaries. An ideal $\mathcal{J}$ is said to be integrally closed if $\mathcal{J}=\overline{\mathcal{J}}$. It is known that radical ideals (in particular prime ideals) are integrally closed, that is, $\sqrt{\mathcal{J}}=\overline{\sqrt{\mathcal{J}}}$. Next we prove a related property that we will need later when proving Theorem 6 .

Lemma 8. For any ideal $\mathcal{J}$, it follows that $\sqrt{\mathcal{J}}=\sqrt{\overline{\mathcal{J}}}$

Proof. Let $\mathcal{J}$ and $\mathcal{K}$ be two ideals such that $\mathcal{J} \subseteq \mathcal{K}$. Then $\overline{\mathcal{J}} \subseteq \overline{\mathcal{K}}$ and $\sqrt{\mathcal{J}} \subseteq \sqrt{\mathcal{K}}$. Also $\overline{\overline{\mathcal{J}}}=\overline{\mathcal{J}}$ and $\sqrt{\sqrt{\mathcal{J}}}=\sqrt{\mathcal{J}}$.

From the definition we have that $\mathcal{J} \subseteq \overline{\mathcal{J}} \subseteq \sqrt{\mathcal{J}}$. Hence taking radicals (that preserve inclusions) we get $\sqrt{\mathcal{J}} \subseteq \sqrt{\overline{\mathcal{J}}} \subseteq \sqrt{\mathcal{J}}$ so that the conclusion of the lemma follows.

3.2. Proof of Theorem 6, From Lemma 8 we have $\sqrt{\mathcal{B}}=\sqrt{\overline{\mathcal{B}}}$. On the other hand, the Hilbert Nullstellensatz tells us that $\mathbf{V}_{\mathbb{C}}(\mathcal{B})=\mathbf{V}_{\mathbb{C}}\left(\mathcal{B}_{j_{\tau}}\right)$ holds if and only if $\sqrt{\mathcal{B}}=\sqrt{\mathcal{B}_{j_{\tau}}}$. Recall that the Hilbert Nullstellensatz works in complex varieties but not in real ones. Combining these two facts we obtain

$$
\overline{\mathcal{B}}_{j_{\tau}} \subseteq \overline{\mathcal{B}} \subseteq \sqrt{\overline{\mathcal{B}}}=\sqrt{\mathcal{B}}=\sqrt{\mathcal{B}_{j_{\tau}}} \subseteq \sqrt{\overline{\mathcal{B}}_{j_{\tau}}}=\overline{\mathcal{B}}_{j_{\tau}}
$$

where in the last step we have used the hypothesis of the theorem. In consequence we get $\overline{\mathcal{B}}=\overline{\mathcal{B}}_{j_{\tau}}$ and therefore $\tau=\kappa$, the reduced Bautin depth of $\mathcal{B}$. The final part of the theorem follows from Theorem 5 .

\section{REFERENCES}

[1] A. F. Andreev, Investigation of the behaviour of the integral curves of a system of two differential equations in the neighbourhood of a singular point, Amer. Math. Soc. Transl. (2) 8 (1958), 183-207. MR0094499 (20 \#1015)

[2] N. N. Bautin, On the number of limit cycles appearing with variation of the coefficients from an equilibrium state of the type of a focus or a center (Russian), Mat. Sbornik N.S. 30(72) (1952), 181-196. MR0045893 (13,652a)

[3] A. Beardon, Complex Analysis: the Winding Number principle in analysis and topology. John Wiley and Sons. 1979

[4] David Cox, John Little, and Donal O'Shea, Ideals, varieties, and algorithms. An introduction to computational algebraic geometry and commutative algebra, 3rd ed., Undergraduate Texts in Mathematics, Springer, New York, 2007. MR2290010 (2007h:13036)

[5] I. A. García, The cyclicity of some degenerate centers with polynomial Bautin ideal, to appear.

[6] Isaac A. García, Jaume Giné, and Maite Grau, A necessary condition in the monodromy problem for analytic differential equations on the plane, J. Symbolic Comput. 41 (2006), no. 9, 943-958, DOI 10.1016/j.jsc.2006.04.007. MR2251812 (2007f:34058) 
[7] I.A. García and D.S. Shafer, Cyclicity of a class of polynomial nilpotent center singularities, to appear.

[8] H. Hauser, J.-J. Risler, and B. Teissier, The reduced Bautin index of planar vector fields, Duke Math. J. 100 (1999), no. 3, 425-445, DOI 10.1215/S0012-7094-99-10015-9. MR.1719738 (2001f:34054)

[9] Craig Huneke and Irena Swanson, Integral closure of ideals, rings, and modules, London Mathematical Society Lecture Note Series, vol. 336, Cambridge University Press, Cambridge, 2006. MR2266432(2008m:13013)

[10] Yulij Ilyashenko and Sergei Yakovenko, Lectures on analytic differential equations, Graduate Studies in Mathematics, vol. 86, American Mathematical Society, Providence, RI, 2008. MR2363178(2009b:34001)

[11] Valery G. Romanovski and Douglas S. Shafer, The center and cyclicity problems: a computational algebra approach, Birkhäuser Boston, Inc., Boston, MA, 2009. MR2500203 (2010h:34001)

[12] Robert Roussarie, Bifurcation of planar vector fields and Hilbert's sixteenth problem, Progress in Mathematics, vol. 164, Birkhäuser Verlag, Basel, 1998. MR.1628014 (99k:58129)

[13] Wolmer Vasconcelos, Integral closure. Rees algebras, multiplicities, algorithms, Springer Monographs in Mathematics, Springer-Verlag, Berlin, 2005. MR2153889 (2006m:13007)

Departament de Matemàtica, Universitat de Lleida, Avda. Jaume II, 69, 25001 Lleida, SPAIN

E-mail address: garcia@matematica.udl.cat 